MATEC Web of Conferences 22,02031 (2015)

DOI: $10.1051 /$ matec conf/ 20152202031

(C) Owned by the authors, published by EDP Sciences, 2015

\title{
A Study on the Storage Reliability of LSINS Based on Step-stress Ac- celerated Life Test
}

\author{
Fei Teng \\ School of Mathematics and Statistics, Beijing Institute of Technology, Beijing, China \\ Yuanyuan Liu \\ Century Finance International Investment Advisory (Beijing), Beijing, China \\ Baosheng Huang \\ School of Mathematics and Statistics, Beijing Institute of Technology, Beijing, China
}

\begin{abstract}
Based on the step-stress accelerated life test and the laser strap-down inertial navigation system, this paper studies the accelerated life model and the test method, provides the likelihood function, the likelihood equation and the two-order derivative when the stress level is $\mathrm{k}$, evaluates the effectiveness of the method with the simulation test model established by MATLAB, applies the research findings in the storage reliability study of the XX laser strap-down inertial navigation system, and puts forward an effective evaluation method of the storage life of the inertial navigation system.
\end{abstract}

Keywords: step-stress accelerated life test; maximum likelihood estimation; MATLAB simulation experiment; storage reliability study

\section{INTRODUCTION}

Laser strap-down inertial navigation system (Hereinafter referred to as the system) is a navigation technology that converts measurement signals transmitted by inertial measurement units (gyroscope and accelerometer) on air vehicles into navigation parameters. The system is an important component of the missile, featuring small volume, light weight, low cost, high precision and so on. The reliability level of the system directly determines the aircraft performance and even the success or failure of a task due to complicated components and more complex battle environment on service. Therefore, the reliability study on the system has become the focus of the system designer and users [1].

Laser gyroscope is the only high-precision inertial sensitive instrument applied practically in the field of inertial technology so far. Featuring quick reaction capability, high reliability and long service life, it is widely used in aviation and aerospace. As an ideal device and an important component of the strap-down inertial navigation system, laser gyroscope percepts changes of the direction of an air vehicle by maintaining the same rotation axis direction of its own so as to send out signals and determine the attitude of the air vehicle. For this reason, the reliability analysis and service life prediction have become an important issue of the reliability study of LSINS. Studies on improving the reliability of gyroscope have been carried out at home and abroad. For example, Rekunov D A et al. introduced a miniature tri-axial laser gyroscope
(TLG)M-40 ${ }^{[2]}$; Kuznetsov A $\mathrm{G}$ et al. took into account the design of LSINS from two precision grades [3]; Yuanzheng Liu et al. studied the reliable ignition technology of laser gyroscope ${ }^{[4]}$. In view of the operating feature of LSINS of long-term storage and one-time usage, the reliability of system storage should attract more and more attention. However, there are only a few methods evaluating the storage life of LSINS effectively. Jing Ma et al. studied the accelerated storage life evaluation based on Brownian motion with drift ${ }^{[5]}$

This paper carries out a study on the evaluation method of the service life of LSINS by designing a step-stress accelerated storage life test and verifies the effectiveness of the method through the MATLAB numerical simulation analysis. Meanwhile, it applies the research findings in the XX laser strap-down inertial navigation system. This method not only can be used in the evaluation of the storage life of LSINS but also provides an effective reference for the service life evaluation of other inertial systems.

\section{PLAN OF THE ACCELERATED LIFE TEST}

Storage reliability refers to products' capability of maintaining required functions in the specified storage conditions as well as the fixed time period ${ }^{[6]}$. The service life of products is improved significantly with the rapid development of modern science and technology. From a practical perspective, the service life of products is usually predicted through the method of 


\section{MATEC Web of Conferences}

accelerated life test, which accelerates the life test with intensified stress conditions (such as temperature, vacuum, voltage and vibration) so as to improve the test efficiency and reduce the test cost.

\subsection{Determination of sensitive stress}

Because products of LSINS are packaged, sensitive stresses mainly include temperature and electric stress Influences of other stresses are relatively weak. Temperature is a major cause of the failure of laser gyroscope in the storage condition. Therefore, temperature is selected as the sensitive stress of laser gyroscope.

\subsection{Test methods}

The test is carried out with grouped data due to practical difficulties of observation methods and test equipment. Constant stress accelerated test is simple in operation, the statistical theory of which is basically mature. The step-stress test accelerates the failure of samples and reduces the number of samples. Stresses in the progressive stress test change fast and become invalid fast as well. The requirements of controlling stress level changes and tracking ineffective equipment are relatively high and the data processing method is not mature yet ${ }^{[7]}$. LSINS is a sophisticated product with a high price and a small sample size. The step-stress test is adopted because the cost of obtaining a higher statistical accuracy would be extremely high. In the meantime, the accelerated stress of the system is related to temperature ${ }^{[8]}$, so the accelerated model of Arrhenius is adopted in the test.

\subsection{Plan of the accelerated life test}

Step-stress tests can be divided into type-I step-stress accelerated life test and type-II step-stress accelerated life test. The type-I step-stress accelerated life test is adopted on account of the high reliability of LSINS, fast products update and short test time. Meanwhile, the failure mechanism might be changed when the extreme temperature exceed $70^{\circ} \mathrm{C}$ in view of the fact that the extreme temperature of LSINS is $70^{\circ} \mathrm{C}$ and the lowest storage temperature is $5^{\circ} \mathrm{C}$. Therefore, the extreme temperature of the test should be lower than $70^{\circ} \mathrm{C}$.

\subsection{Accelerated test plan}

After the usage or the storage of LSINS for a period of time, output signals and the intensity might be reduced gradually. Failure criteria are determined in accordance with the requirements of system performance. In the temperature test with the same stress, tests should be conducted as many as possible. For example, the inertial navigation system is recovered to a normal temperature state at a fixed moment every 3 to 5 days. Tests of output signals and the intensity are carried out with detailed data recording on the premise that operating parameters of the inertial navigation system remain unchanged.
3 GROUPED DATA STATISTICS AND NUMERICAL SIMULATION ANALYSIS OF THE STEP-STRESS ACCELERATED LIFE TEST

A group of accelerated stress levels is selected in the step-stress accelerated life test. Suppose there are $k$ stress levels, such as $S_{1}, S_{2}, \ldots, S_{k}$, a batch of products is tested with the accelerated stress $S_{1}$. The stress level is increased to $S_{2}$ after the test is maintained for a certain time period. Continue the test for products that are not invalid with the accelerated stress $S_{2}$ and stop the test after the maximum stress $s_{k}$ is maintained for a certain time period ${ }^{[9]}$.

\subsection{Basic assumptions}

(1) In the case that the stress level is $S_{\mathrm{i}}$, the service life of products obeys the exponential distribution with a failure rate of $\lambda_{i}$, that is, $F_{i}(t)=1-\exp \left(-\lambda_{i} t\right)$ $(i=1,2, \ldots, k)$.

(2) Select $n$ samples randomly from a batch of products for the step-stress accelerated life test. The censored time is $\tau_{i}$ and the observation frequency is $q_{i}$ when the stress level is $S_{\mathrm{i}}$. The observed failure interval is:

$\left[t_{i 1}^{\prime}, t_{i 1}\right], \ldots,\left[t_{i j}^{\prime}, t_{i j}\right], \ldots,\left[t_{i q_{i}}^{\prime}, t_{i q_{i}}\right] i=$

$1,2, \ldots, k, j=1,2, \ldots q_{i}$

(3) When the stress level is $S_{\mathrm{i}}$, the number of failed

products is $r_{i}$ and the number of failed products

observed in the failure interval of $\left[t_{i j}^{\prime}, t_{i j}\right]$ is $r_{i j}$.

The two satisfy $r_{i}=\sum_{j=1}^{q_{i}} r_{i j}$. The accumulated total of failures is $Z=\sum_{i=1}^{k} r_{i} \leq n$ and the number of products that are not failed is $n-Z$.

(4) The remaining life of products only depends on the accumulated failure part and the current stress level, which has nothing to do with the cumulative way. Data conversion can be carried out in line with the famous cumulative exposure model proposed by Nelson (1980) ${ }^{[10]}$. The conversion formula is $t_{i}=\frac{\lambda_{j}}{\lambda_{i}} t_{j}$. The step-stress accelerated life test is compensated according to the formula so as to obtain the real life data, $\tilde{t}_{i j}^{\prime}, \tilde{t}_{i j}, \tilde{\tau}_{i}$. 
$\tilde{\tau}_{i}=\sum_{m=1}^{i-1} \frac{\lambda_{m}}{\lambda_{i}} \tau_{m}+\tau_{i}=\sum_{m=1}^{i} \frac{\lambda_{m}}{\lambda_{i}} \tau_{m}$

$\tilde{t}_{1 j}^{\prime}=\left(\tilde{\tau}_{i}-\tau_{i}\right)+t_{1 j}^{\prime}, \tilde{t}_{1 j}=\left(\tilde{\tau}_{i}-\tau_{i}\right)+t_{1 j}$

$\tilde{t}_{i j}^{\prime}=\tilde{\tau}_{i-1} \cdot \frac{\lambda_{i-1}}{\lambda_{i}}+t_{i j}^{\prime}=\sum_{m=1}^{i-1} \frac{\lambda_{m}}{\lambda_{i}} \tau_{m}+t_{i j}^{\prime}$

$\tilde{t}_{i j}=\tilde{\tau}_{i-1} \cdot \frac{\lambda_{i-1}}{\lambda_{i}}+t_{i j}=\sum_{m=1}^{i-1} \frac{\lambda_{m}}{\lambda_{i}} \tau_{m}+t_{i j}$

3.2 Maximum likelihood estimation of the step-stress accelerated life test ${ }^{[1]]}$

(1) According to the assumptions mentioned above and the definition of likelihood function, the equation of likelihood function and the log-likelihood function can be obtained:

$L=E \cdot \exp \left(-\lambda_{k} \tilde{\tau}_{k}(n-Z)\right) \cdot$

$\prod_{i=1}^{k} \prod_{j=1}^{q_{i}}\left\{\left[\exp \left(-\lambda_{i} \cdot \tilde{t}_{i j}^{\prime}\right)-\exp \left(-\lambda_{i} \cdot \tilde{t}_{i j}\right)\right]\right\}^{r_{i j}}$

$\tilde{L}=\ln L=\ln E-\lambda_{k} \tilde{\tau}_{k}(n-Z)$

$+\sum_{i=1}^{k} \sum_{j=1}^{q_{i}} r_{i j} \cdot \ln \left[\exp \left(-\lambda_{i} \cdot \tilde{t}_{i j}^{\prime}\right)-\exp \left(-\lambda_{i} \cdot \tilde{t}_{i j}\right)\right]$

where $E=\frac{r_{i} !}{r_{i 1} ! r_{i 2} ! \ldots r_{i q_{i}} !}$

(2) Because the real life data $\tilde{t}_{i j}^{\prime}, \tilde{t}_{i j}, \tilde{\tau}_{i}$ cannot be obtained from the test directly, it is substituted by test data $t_{i j}^{\prime}, t_{i j}, \tau_{i}$. In the meantime, Arrhenius model is selected for the service life, $\ln \left(\frac{1}{\lambda_{i}}\right)=\alpha+\frac{\beta}{S_{i}}(i=1,2, \ldots, k) \quad, \quad$ that $\quad$ is $\lambda_{i}=\exp \left(-\alpha-\beta / S_{i}\right)$. The log-likelihood function can be transformed as:
$\tilde{L}=\ln E-\sum_{m=1}^{k} \tau_{m} \cdot \exp \left(-\alpha-\beta / S_{m}\right)(n-Z)+\sum_{i=1}^{k} \sum_{m=1}^{i-1} r_{i} \cdot \tau_{m} \cdot \exp \left(-\alpha-\beta / S_{m}\right)$ $+\sum_{i=1}^{k} \sum_{j=1}^{q} r_{i j} \cdot \ln \left\{\exp \left[-t_{i j}^{\prime} \cdot \exp \left(-\alpha-\beta / S_{i}\right)\right]-\exp \left[-t_{i j} \cdot \exp \left(-\alpha-\beta / S_{i}\right)\right\}\right.$

In the formula above, $\alpha, \beta$ are parameters to be estimated and the maximum likelihood estimations are $\hat{\alpha}, \hat{\beta}$. The point estimation of the failure rate under the normal stress level of $S_{0}$ is: $\hat{\lambda}_{0}=\exp \left(-\hat{\alpha}-\hat{\beta} / \mathrm{S}_{0}\right)$.

(3) Solve the derivatives of $\alpha, \beta$ with the log-likelihood function above:

$$
\left\{\begin{array}{l}
a_{i}=\exp \left(-\alpha-\beta / S_{i}\right) \\
a_{m}=\exp \left(-\alpha-\beta / S_{m}\right) \\
b=\exp \left(-t_{i j}^{\prime} \cdot \exp \left(-\alpha-\beta / S_{i}\right)\right) \\
c=\exp \left(-t_{i j} \cdot \exp \left(-\alpha-\beta / S_{i}\right)\right)
\end{array}\right.
$$

The likelihood equation is obtained as follows:

$$
\left\{\begin{array}{l}
\frac{\partial \tilde{L}}{\partial \alpha}=\sum_{m=1}^{k} \tau_{m} \cdot a_{m}(n-\mathrm{Z})+\sum_{i=1}^{k} \sum_{m=1}^{i-1} r_{i} \cdot \tau_{m} \cdot a_{m} \\
+\sum_{i=1}^{k} \sum_{j=1}^{q} r_{i j} \cdot a_{i} \frac{t_{i j}^{\prime} \cdot b-t_{i j} \cdot c}{b-c} \\
\frac{\partial \tilde{L}}{\partial \beta}=\sum_{m=1}^{k} \frac{\tau_{m} \cdot a_{m}(n-\mathrm{Z})}{S_{m}}+\sum_{i=1}^{k} \sum_{m=1}^{i-1} \frac{r_{i} \cdot \tau_{m} \cdot a_{m}}{S_{m}} \\
+\sum_{i=1}^{k} \sum_{j=1}^{q} \frac{r_{i j} \cdot a_{i}}{S_{i}} \cdot \frac{t_{i j}^{\prime} \cdot b-t_{i j} \cdot c}{b-c}
\end{array}\right.
$$

Two-order derivatives of the log-likelihood function about $\alpha, \beta$ are shown below:

$\frac{\partial^{2} \tilde{L}}{\partial \alpha^{2}}=-\sum_{m=1}^{k} \tau_{m} \cdot a_{m}(n-\mathrm{Z})-\sum_{i=1}^{k} \sum_{m=1}^{i-1} r_{i} \cdot \tau_{m} \cdot a_{m}$

$-\sum_{i=1}^{k} \sum_{j=1}^{q_{i}} r_{i j} \cdot\left[\frac{a_{i}}{b-c} \cdot\left(t_{i j}^{\prime} \cdot \mathrm{b}-t_{i j} \cdot \mathrm{c}\right)+\frac{a_{i}^{2} b c}{(b-c)^{2}} \cdot\left(t_{i j}^{\prime}-t_{i j}\right)^{2}\right]$

$\frac{\partial^{2} \tilde{L}}{\partial \beta^{2}}=-\sum_{m=1}^{k} \frac{\tau_{m} \cdot a_{m}(n-Z)}{S_{m}{ }^{2}}-\sum_{i=1}^{k} \sum_{m=1}^{i-1} \frac{r_{i} \cdot \tau_{m} \cdot a_{m}}{S_{m}{ }^{2}}$

$-\sum_{i=1}^{k} \sum_{j=1}^{q_{i}} \frac{r_{i j}}{S_{i}^{2}} \cdot\left[\frac{a_{i}}{b-c} \cdot\left(t_{i j}^{\prime} \cdot \mathrm{b}-t_{i j} \cdot \mathrm{c}\right)+\frac{a_{i}^{2} b c}{(b-c)^{2}} \cdot\left(t_{i j}^{\prime}-t_{i j}\right)^{2}\right]$ 


\section{MATEC Web of Conferences}

Table 1. Simulation results when the benchmark life of the step-stress accelerated life test with the simple stress Arrhenius model is 2000

\begin{tabular}{|l|l|l|l|l|l|l|}
\hline \multicolumn{7}{|c|}{ Step-stress ArrheniusMain(-9.5,18.2,2000,n,[1.1 1.15 1.17 1.2 ],2000) } \\
\hline $\mathrm{n}$ & $\mathrm{a}=-9.5$ & $\mathrm{~b}=18.2$ & \multicolumn{2}{l|}{ realMeanlife=6002.91 } \\
\hline 40 & mean_A & std_A & mean_B & std_B & medLife & No solution \\
\hline 120 & -9.66744 & 7.435181 & 18.74142 & 8.164852 & 6572.944 & 1 \\
\hline 200 & -9.65113 & 4.450006 & 18.50832 & 5.015491 & 6190.026 & 1 \\
\hline 400 & -9.39943 & 3.206062 & 18.15422 & 3.619161 & 5971.978 & 0 \\
\hline 600 & -9.3933 & 2.761696 & 18.10882 & 3.094462 & 5930.844 & 0 \\
\hline 800 & -9.47337 & 2.16044 & 18.21122 & 2.494359 & 6001.272 & 1 \\
\hline 1000 & -9.56101 & 1.449487 & 18.29703 & 1.843004 & 6040.551 & 0 \\
\hline 1200 & -9.57734 & 1.398243 & 18.32999 & 2.078428 & 6049.296 & 0 \\
\hline
\end{tabular}

Table 2. Simulation results when the benchmark life of the step-stress accelerated life test with the simple stress Arrhenius model is 3000

\begin{tabular}{|l|l|l|l|l|l|l|}
\hline \multicolumn{7}{|c|}{ Step-stress ArrheniusMain(-9.5,18.2,2000,n,[1.1 1.15 1.17 1.2 ],2000) } \\
\hline $\mathrm{n}$ & \multicolumn{2}{|l|}{$\mathrm{a}=-9.5$} & $\mathrm{~b}=18.2$ & \multicolumn{2}{l|}{ realMeanlife=6002.91 } \\
\hline 40 & mean_A & std_A & mean_B & std_B & medLife & No solution \\
\hline 120 & -9.98676 & 5.999628 & 18.81068 & 6.752799 & 6449.605 & 0 \\
\hline 200 & -9.5389 & 3.72548 & 18.27331 & 4.197428 & 6097.773 & 1 \\
\hline 400 & -9.56587 & 2.884238 & 18.2849 & 3.32562 & 6012.895 & 0 \\
\hline 600 & -9.48439 & 1.715998 & 18.19745 & 1.948324 & 6020.493 & 0 \\
\hline 800 & -9.55065 & 1.951269 & 18.20376 & 2.03834 & 5993.912 & 0 \\
\hline 1000 & -9.52397 & 1.293215 & 18.27643 & 1.745232 & 6025.503 & 1 \\
\hline 1200 & -9.51529 & 1.003901 & 18.21766 & 1.139009 & 6016.44 & 1 \\
\hline
\end{tabular}

$\frac{\partial^{2} \tilde{L}}{\partial \alpha \partial \beta}=-\sum_{m=1}^{k} \frac{\tau_{m} \cdot a_{m}(n-Z)}{S_{m}}-\sum_{i=1}^{k} \sum_{m=1}^{i-1} \frac{r_{i} \cdot \tau_{m} \cdot a_{m}}{S_{m}}$

$-\sum_{i=1}^{k} \sum_{j=1}^{q_{i}} \frac{r_{i j}}{S_{i}} \cdot\left[\frac{a_{i}}{b-c} \cdot\left(t_{i j}^{\prime} \cdot \mathrm{b}-t_{i j} \cdot \mathrm{c}\right)+\frac{a_{i}^{2} b c}{(b-c)^{2}} \cdot\left(t_{i j}^{\prime}-t_{i j}\right)^{2}\right]$

3.3 Numerical simulation of the step-stress accelerated life test

The log-likelihood function is optimized comprehensively with the built-in programming of MATLAB for the solution of extremal function. Simulate $\alpha, \beta$ with given truth-values, that is, $\theta_{0}=\exp (\hat{\alpha}+\hat{\beta})$, with the method of generating exponential random numbers so as to verify the effectiveness of the method mentioned above.

\subsubsection{Numerical simulation conditions}

The programming command is: ArrheniusMain (realA, realB, realtfo, $\mathrm{n}$, realf, $\mathrm{mm}$ )

(1) realA and realB are the truth-values of parame- ters to be estimated $\alpha, \beta$. According to the real mean life of a living example, realMeanlife $=6002.91$, and it can be solved that $\alpha+\beta=8.7$.

(2) realf is the test stress vector: it is selected as [1.1 $1.151 .21 .17]$ according to the temperature ratio of a living example.

(3) $\mathrm{mm}$ is the number of simulation times: 2000 times.

(4) Carry out the numerical simulation with vibrations of the sample size $n$ and the life realtf0 under the benchmark stress.

\subsubsection{Simulation results}

It can be concluded from Table 1 and Table 2 : simulation results of parameters to be estimated $\alpha, \beta$ and truth-values are extremely close when the sample size $\mathrm{n}$ is large enough. The storage life estimation is also close to the real life with higher estimated accuracy when the standard deviation is small. It indicates that the way of evaluating the service life of products with the maximum likelihood estimation of step-stress accelerated life test is quite effective. 
ICETA 2015

Table 3. Storage time of 10 gyroscopes under the stress $S_{1}$ (storage temperature) (Unit: day)

\begin{tabular}{|l|l|l|l|l|l|l|l|l|l|l|}
\hline Gyro No. & 1 & 2 & 3 & 4 & 5 & 6 & 7 & 8 & 9 & 10 \\
\hline $\begin{array}{l}\text { Production } \\
\text { date }\end{array}$ & $9-5$ & $4-9$ & $5-14$ & $7-11$ & $7-11$ & $9-5$ & $12-30$ & $12-30$ & $12-30$ & $12-30$ \\
\hline Storage time & 623 & 772 & 737 & 679 & 679 & 623 & 507 & 507 & 507 & 507 \\
\hline
\end{tabular}

Table 4. Test time and the number of failures under different stress levels

\begin{tabular}{|l|l|l|l|l|l|l|l|}
\hline Stress level & $\mathrm{S}_{2}$ & $\mathrm{~S}_{3}$ & $\mathrm{~S}_{4}$ & $\mathrm{~S}_{5}$ & $\mathrm{~S}_{6}$ & $\mathrm{~S}_{7}$ & $\mathrm{~S}_{8}$ \\
\hline Test time $\tau$ & 20 & 20 & 20 & 40 & 10 & 20 & 40 \\
\hline Number of failures $\gamma$ & 0 & 0 & 0 & 1 & 0 & 0 & 0 \\
\hline
\end{tabular}

\section{STUDY ON THE STORAGE RELIABILITY OF} A CERTAIN LSINS

$\mathrm{XX}$ LSINS is expensive and the storage reliability research has strict requirements on storage test samples, service trials cannot be provided after the production. The undertaker is unable to provide suitable LSINS but only 10 laser gyroscopes for the storage test. In view of the fact that gyroscope is an important component and a weak link of LSINS as well as a core problem of the study on the storage reliability of LSINS, the system storage life evaluation is carried out with gyroscopes as the storage sample.

\subsection{Data of accelerated storage life test}

Select 10 gyroscopes to carry out the step-stress accelerated life test, of which the stress is temperature $\left({ }^{\circ} \mathrm{C}\right)$. The first level stress is $20^{\circ} \mathrm{C}$, which is also the storage temperature. The start time is the manufacturing time, which is 2007. The end time is the start time of the accelerated life test, which is May 20, 2009. There are altogether 8 stress levels:

$S_{1}=20, S_{2}=5, S_{3}=40, S_{4}=50$

$S_{5}=55, S_{6}=60, S_{7}=50, S_{8}=20$

Refer to Table 3

and Table 4 for specific data (Gyro No.1 becomes invalid on the $40^{\text {th }}$ day under the stress level of $S_{5}$ ).

\subsection{Maximum likelihood estimation}

(1) The fault time (converted) of the faulty product under the $\mathrm{k}^{\text {th }}$ stress can be expressed as:

$z_{1}=\frac{\theta_{k}}{\theta_{1}} D_{1}+\frac{\theta_{k}}{\theta_{2}} \tau_{2}+\frac{\theta_{k}}{\theta_{3}} \tau_{3}+\frac{\theta_{k}}{\theta_{4}} \tau_{4}+\frac{\theta_{k}}{\theta_{5}} \tau_{5}$

Here, $D_{1}=623, \tau_{5}=40$

The converted censored time of products No.2, $3, \ldots \ldots, 10$ under the $\mathrm{k}^{\text {th }}$ stress should be:

$\tau_{i}^{\prime}=\frac{\theta_{k}}{\theta_{1}} D_{i}+\sum_{j=2}^{k} \frac{\theta_{k}}{\theta_{j}} \tau_{j}, \quad i=2,3, \cdots, 10$
(2) The likelihood function of

$$
x_{i}=1000 /\left(237.16+S_{i}\right) \text { is: }
$$

$\ln (L)=-\left(\alpha+\beta x_{5}\right)-\sum_{i=1}^{10} D_{i} \cdot \exp \left(-\alpha-\beta x_{1}\right)$

$-\sum_{i=2}^{k} \tau_{i} \cdot \exp \left(-\alpha-\beta x_{i}\right)$

(3) Partial derivative of $\alpha, \beta$

$$
\left\{\begin{array}{l}
\frac{\partial \ln L}{\partial \alpha}=-1+\left[\sum_{i=1}^{10} D_{i} \exp \left(-\alpha-\beta x_{1}\right)\right. \\
\left.+\sum_{i=2}^{k} \tau_{i} \exp \left(-\alpha-\beta x_{i}\right)\right]=0 \\
\frac{\partial \ln L}{\partial \beta}=-x_{5}+\left[\sum_{i=1}^{10} D_{i} x_{i} \exp \left(-\alpha-\beta x_{1}\right)\right. \\
\left.+\sum_{i=2}^{k} \tau_{i} x_{i} \exp \left(-\alpha-\beta x_{i}\right)\right]=0
\end{array}\right.
$$

(4) Calculate the two-order derivative

$$
\left\{\begin{array}{l}
\frac{\partial^{2} \ln L}{\partial \alpha^{2}}=-\left[\sum_{i=1}^{10} D_{i} \exp \left(-\alpha-\beta x_{1}\right)\right. \\
\left.+\sum_{i=2}^{k} \tau_{i} \exp \left(-\alpha-\beta x_{i}\right)\right] \\
\frac{\partial^{2} \ln L}{\partial \beta^{2}}=-\left[\sum_{i=1}^{10} D_{i}\left(x_{i}\right)^{2} \exp \left(-\alpha-\beta x_{1}\right)\right. \\
\left.+\sum_{i=2}^{k} \tau_{i}\left(x_{i}\right)^{2} \exp \left(-\alpha-\beta x_{i}\right)\right] \\
\frac{\partial^{2} \ln L}{\partial \alpha \partial \beta}=\frac{\partial^{2} \ln L}{\partial \beta \partial \alpha}=-\left[\sum_{i=1}^{10} D_{i} x_{i} \exp \left(-\alpha-\beta x_{1}\right)\right. \\
\left.+\sum_{i=2}^{k} \tau_{i} x_{i} \exp \left(-\alpha-\beta x_{i}\right)\right]
\end{array}\right.
$$




\section{MATEC Web of Conferences}

(5) Use the Newton method to iterate the case and solve the values of $\alpha, \beta: \alpha=-54.1192$, $\beta=19.9484$

\subsection{Storage life evaluation results of gyroscope}

According to the reliability function $R(t)=P(T>t)=1-F(t)=\exp (-\lambda t)$

reliability indexes can be obtained as follows:

Scheme 1: When gyroscopes are stored in the indoor temperature $\left(20^{\circ} \mathrm{C}\right)$, reliability indexes are:

(1) When the storage life is longer than 15 years, the probability is $99.52 \%$;

(2) When the storage life is longer than 10 years, the probability is $99.68 \%$;

(3) When the reliability level is $99.9 \%$, the reliable storage life is 3.1 years;

(4) When the reliability level is $99 \%$, the reliable storage life is 31.2 years.

Scheme 2: Storage environment has experienced four seasons. Here, temperatures of four seasons (take the higher temperature of each season) are: $[5,20,33$, 20]. After the conversion of accelerated factors, reliability indexes of gyroscopes in temperature conversion of four seasons are:

(1) When the storage life is longer than 15 years, the probability is $97.61 \%$;

(2) When the storage life is longer than 10 years, the probability is $98.40 \%$;

(3) When the reliability level is $99 \%$, the reliable storage life is 6.2 years;

(4) When the reliability level is $95 \%$, the reliable storage life is 31.8 years;

(5) When the reliability level is $90 \%$, the reliable storage life is 65.4 years;

\section{CONCLUSION}

This paper studies the accelerated life model that is suitable for LSINS, designs a highly-efficient step-stress accelerated test method, and provides the likelihood function, the likelihood equation and the two-order derivative when the stress level of the step-stress accelerated life test is k. It builds up a simulation test model with MATLAB. The result indicates that the estimated standard deviation of the truth-value is small and the precision is high when the sample size is enough. The effectiveness of the system storage life is verified through the maximum likelihood estimation. Andit evaluates the method of system storage life and provides the method of estimating the probability that the system is stored to the predetermined year based on the data of the accelerated life test of a laser gyroscope.

\section{REFERENCES}

[1] Ren, Z.Q. 2008. The reliability enhancement test technology of LSINS, Electronic Product Reliability and Environmental Testing, (1)

[2] Rekunov D A, Chirkov V A, Uspekhov N V. 2011. Small three-axis laser gyroscope M-40, Gyroscopy and Navigation, 2(4): 222-228.

[3] KuznetsovA G, Portnov B I, Izmailov E A. 2014. Two classes of aircraft strapdown inertial navigation systems on laser gyros: Development and test results. Gyroscopy and Navigation, 5(4): 187-194.

[4] Liu, Y.Z., Wang, J.L., Xue, X.Q. \& Tao, B.L. 2013. Techniques to facilitate laser gyroscope ionization, Journal of Chinese Inertial Technology, (5): 656-659

[5] Ma, J., Yuan, D.D., Chao, D.H. \& Chen, S.Y. 2010. Accelerated storage life assessment of fiber-optic gyroscope based on Brownian motion with drift, Journal of Chinese Inertial Technology, (6)

[6] QJ 3250-2005. Guideline of Non-operating Reliability Design and Evaluation for Space Products [S], 2005.

[7] Qi, L. \& Pan, T. 2014. A comprehensive review of accelerated life test, Science and Technology of China, (20): 90-91

[8] Zhang, F. 2006. A Study on the Accelerated Life Test of Gyroscope, Nanjing University of Science and Technology.

[9] Mao, S.S. \& Wang, L.L. 1997. Accelerated Life Test, Beijing Science Press

[10]Nelson W B. 1980. Accelerated life testing: step-stress models and data analysis, IEEE Transactions on Reliability, 29(2): 103-108.

[11]Cheng, J.X. \& Ming, J. 2004. Analysis of grouped and censored data from step-stress life test, IEEE Transactions on Reliability, 53(1): 22-28. 